\title{
Tensiones de un centro comunitario: análisis desde la psicología comunitaria en Chile
}

\section{Tensions of a Community Center: Analysis based on Community Psychology in Chile}

\author{
Claudia Carrasco-Aguilar ${ }^{\mathrm{a}}$, Gustavo Cerda-Gonzalez ${ }^{\mathrm{b}}$, María Julia Baltar-de Andradec \\ Cristian Zamora-Astudilloa ${ }^{a}$ \& Álvaro Castillo-Muñoz ${ }^{\mathrm{a}}$ \\ ${ }^{a}$ Universidad de Playa Ancha, Valparaíso, Chile \\ ${ }^{b}$ Centro de Pensamiento y Acción Crítica de Valparaíso, Valparaíso, Chile \\ ${ }^{c}$ Pontificia Universidad Católica de Valparaíso, Valparaíso, Chile
}

\begin{abstract}
Resumen: A través de un estudio de caso con base en observaciones, entrevistas y un grupo focal, este artículo describe las tensiones de una organización colectiva que se independiza de una organización no gubernamental en un emergente centro comunitario de la Región de Valparaíso de Chile (quinta región); relacionando dichas tensiones a la subjetividad de psicólogo comunitario que se construye en tal contexto. Los resultados muestran una primera tensión disciplinaria, que se refiere al papel de la psicología en el centro comunitario, y una segunda tensión política, que analiza la función social del centro y el voluntariado como mecanismo para esa función.
\end{abstract}

Palabras clave: psicología comunitaria, centro comunitario, tensiones.
Abstract: Through a study case based on observations, interviews and a focus group, this article seeks to describe the tensions that arise in a collective organization, which becomes independent of a non-government organization in an emerging community center in the region of Valparaiso, Chile (fifth region). This tensions are related to the subjectivity of the community psychologist which is constructed in said context. The results show an initial disciplinary tension, which refers to the role of the psychology in the community center and a second political tension, which analyzes the social function of the community center and the volunteer work as mechanism for that function.

Keywords: community psychology, community center, tensions.

Contacto: C. Carrasco Aguilar. Independencia 2002, Valparaíso, Quinta Región, Chile. Correo electrónico: claudia.carrasco@upla.cl

Cómo citar: Carrasco-Aguilar, C., Cerda-Gonzalez, G., Baltar-de Andrade, M. J., Zamora-Astudillo, C., \& Castillo-Muñoz, A. (2016). Tensiones de un centro comunitario: análisis desde la psicología comunitaria en Chile. Revista de Psicología, 25(1), 1-22.

http://dx.doi.org/10.5354/0719-0581.2016.41459 


\section{Introducción}

\section{El papel del Estado en la psicología co- munitaria en Chile}

Según Ussher (2006), en la segunda mitad del siglo XX la psicología comunitaria se delinea como una rama de la psicología social, nutriéndose de las prácticas de psicólogos y psicólogas que actuaban tratando de dar respuesta a realidades de exclusión social, pobreza y violencia. La década de los 60 fue clave en esta disciplina (Ornelas, 1997), la psicología comunitaria comenzó a focalizarse en el trabajo con poblaciones socialmente marginadas, con un sentido de facilitar la participación.

Para Berroeta Torres, Hatibovich Diaz y Asún Salazar (2012), en Chile la psicología comunitaria ha atravesado diferentes momentos, dependiendo del contexto sociopolítico y los respectivos marcos institucionales derivados de las políticas públicas. En la década de los 80, "la intervención se realiza principalmente desde las Organizaciones No Gubernamentales (ONG) en prácticas fuera de la institucionalidad gubernamental y centradas principalmente en la lucha por la reconstrucción democrática" (p. 336).

Para Oxhorn (2004), esto coincidiría con el devenir político y social, ya que en Chile solo a finales de los años 70 los sectores populares se convirtieron en actores sociales autónomos, con independencia de los partidos políticos, lo que ocurrió en un contexto social de dictadura militar. Dichos movimientos se organizaron en torno a la lucha por la democracia y condiciones de vida más dignas.

En países como Brasil (dos Santos Azevêdo, 2009), el auge e inicio de la psicología comunitaria coincide, al igual que en Chile, con el periodo de dictadura mili- tar, lo que podría indicar que corresponde a un fenómeno internacional. Posteriormente, las prácticas de la psicología comunitaria en los 90 se incorporaron de manera progresiva a la nueva institucionalidad gubernamental de los primeros gobiernos democráticos, convirtiendo a las $\mathrm{ONG}$, en ejecutoras de las políticas del Estado (Berroeta Torres et al., 2012). Es así como en esa década las organizaciones comenzaron a buscar estrategias para proyectar las prácticas generadas en la exclusión, en una sociedad que priorizaba la democracia y la creación de institucionalidad con mecanismos y/o recursos en función de la participación (Bustos Titus, 1996).

Para Oxhorn (2004), estos intentos fracasaron, al menos parcialmente, por la debilidad del actor social popular luego de la dictadura, lo que fundamenta en la idea de la paradoja del gobierno autoritario "la presencia de un gobierno electo significó que ya no había un 'enemigo' claro contra el cual unificar a los heterogéneos sectores populares" (p. 76). Esto debe ser analizado junto con las nuevas subjetividades construidas, bajo las cuales se fue perdiendo lo que Paredes (2013) entiende por el sentido de agencia, es decir "la capacidad de generar la consciencia de que es posible cambiar las prácticas sociales que producen la situación injusta, a través de la acción colectiva y la movilización" (p. 22), poniendo en tensión el papel original de la psicología comunitaria. Esto introdujo múltiples tensiones respecto del sentido y finalidad de las intervenciones llevadas a cabo por psicólogos en contextos comunitarios, ya que incluso los lugares de trabajo en los que se desempeñaban estaban reconvirtiéndose, producto de la pérdida del financiamiento externo, hacia los intereses del Estado (Berroeta Torres et al., 2012).

El problema del intervencionismo del Estado en las ONG no fue solo paradojal, 
sino también laboral y de sentido. Estas organizaciones terminaron tercerizando la labor del Estado y convirtiendo a los psicólogos comunitarios en subcontratados estatales, bajo figuras contractuales que invisibilizaban esta relación. Tal como señala Alfaro Inzunza (2007), se verificó un paulatino proceso de institucionalización disciplinar en la psicología comunitaria, que puso a Chile como un caso especial en el marco latinoamericano, en cuanto ya existía una importante distancia entre el quehacer de esta disciplina y los principios que conformaban el paradigma de la transformación social. Para Alfaro Inzunza y Zambrano Constanzo (2009), la inserción de psicólogos y psicólogas en programas sociales, derivados de políticas estatales, terminó por refundar la psicología comunitaria nacional.

Posteriormente, la tensión a la que se comienzan a ver enfrentados los psicólogos se vinculó con los intereses detrás de sus acciones y, tal como señala Bustos Titus (1996), el desafío estuvo en actuar desde la institucionalidad con una lógica que se había construido en los márgenes de la misma. Las ONG transitaron, en las dos últimas décadas, desde focalizarse en los intereses de la sociedad civil apoyados por el financiamiento extranjero, hasta convertirse en instituciones que implementan la política del Estado. Para Díez Rodríguez (1999), las ONG se habían vuelto necesarias para los sistemas neoliberales, ya que aportaban a la rearticulación social que necesita el modelo neoliberal para paliar los costos de las políticas, ya sea atendiendo a los casos más extremos o canalizando la reivindicación social a través de la participación solidaria.

Sin embargo, en Chile las ONG han ido cumpliendo paulatinamente la función de implementación de las políticas del Esta- do, perdiendo incluso dicha función. A esto se le suma lo que señalan Berroeta Torres et al. (2012), quienes afirman que durante el 2000 se consolida y tecnifica por parte del Estado "un enfoque de habilitación individual en el abordaje de los problemas y la integración social, dejando en segundo plano las iniciativas de tipo territorial" (p. 2).

\section{Las posibilidades de una psicología comunitaria crítica}

Pese al enfoque individual de los fenómenos sociales promovidos por el Estado, diversas formas de organización social han prevalecido. Para Asún y Zúñiga (2013), estas han estado más bien vinculadas con las protestas sociales, las que operan como parte constitutiva de democracias, a la vez que expresan el debilitamiento de otras formas tradicionales de participación política, como la electoral, la militancia partidista y el involucramiento en campañas.

Entonces, es posible afirmar que el descontento social de hace décadas no ha terminado, aunque las formas de expresarlo han cambiado desde la Dictadura, manteniendo la desconfianza en las instituciones y en el papel del Estado. Tal como afirma Fernández (2013), desde hace algunos años han comenzado a instalarse en el discurso público diversas expresiones que simbolizan el deseo de profundización democrática y de ampliación de la participación ciudadana, las cuales reflejarían descontento social, así como la necesidad de recuperar la ciudadanía. Con estas transformaciones en las formas y sentidos de la organización social, cabe preguntarse cuál ha sido el papel de los psicólogos comunitarios; y si es posible la construcción de una subjetividad que logre, en el actual escenario social, llevar a cabo intervenciones que 
sigan colocando en el centro la participación social.

Para Montero (2008), los principios de la psicología comunitaria se encuentran contenidos en lo que denomina el paradigma de la construcción y transformación crítica. La psicología comunitaria se constituye desde sus inicios con claras orientaciones de cómo deben abordarse los problemas psicosociales, explicitando los procesos políticos vinculados con la reproducción de las injusticias sociales, así como buscando la construcción transformadora de las comunidades más pobres y marginadas (Montero, 2003).

Para dos Santos Azevêdo (2009), en Latinoamérica la psicología comunitaria debe instalar una práctica reivindicativa hacia acciones políticas que sitúen un modelo de actuación para los psicólogos frente a los problemas sociales, con una visión crítica y de compromiso social. Sin embargo, estos principios son complejos de ser evidenciados en una práctica de psicólogos comunitarios con un fuerte trabajo institucionalizado, como es el caso de Chile (Alfaro Inzunza \& Zambrano Constanzo, 2009; Asún \& Unger, 2007)

\section{La subjetividad del psicólogo/a comu- nitario/a}

La institucionalización del trabajo de la psicología comunitaria en Chile implicaría ciertos lineamientos para el marco de acción de los psicólogos comunitarios. Si se sigue a Berroeta Torres et al. (2012), y se asume que existe un enfoque de habilitación individual en el abordaje de los problemas y la integración social, se estaría frente a un modo de objetivación que, según Foucault (1983), convierte a los seres humanos en sujetos, cuestionando el rango de individuos de la categoría psicólogo comunitario.
Para Foucault (1983), esta forma de poder interviene en la inmediatez de la vida cotidiana que categoriza al individuo, marcándolo con su propia individualidad y, de algún modo, termina por atarlo a su propia identidad. Al realizar esto último, este poder le impone una ley de verdad que debe reconocer y que los demás han de reconocer en él, tratándose de una forma de dominio que hace sujetos individuales: psicólogos comunitarios sujetos a la política del Estado, actuando en favor de los marginados (Berroeta Torres et. al., 2012).

Y es que para Foucault (1983), existen al menos dos significados de la palabra sujeto: sujeto a algún otro mediante el control y la dependencia, y sujeto y atado a su propia identidad por una conciencia o por el conocimiento de sí. Ambos sentidos sugieren una forma de poder que subyuga y sujeta $\mathrm{y}$, también en ambos casos, se evidencia el poder político del Estado, como forma de dominio que es tanto individualizadora como totalizadora. Para el autor, nunca ha habido una combinación tan intrincada de las mismas estructuras de técnicas de individuación y de procedimientos de totalización, como en la noción de subjetividad.

Lo que generaría el éxito de esta forma de poder es, precisamente, el hecho de que el Estado moderno no es una entidad que se ha desarrollado por encima de los individuos o que ignora lo que son, sino por el contrario, se trataría de una estructura muy sofisticada, en la que se pueden integrar los individuos con la condición de que esta individualidad adopte una nueva forma y se someta a un conjunto de dispositivos muy específicos.

Sin embargo, siguiendo a Pérez (2002), es necesario señalar que no se trata en este análisis de proponer un orden causal. La sociedad moderna no fue como fue gra- 
cias a esta subjetividad, o al revés, ya que una y otra deben ser entendidas como construcciones correspondientes y correspondientemente necesarias.

Entonces, no se trata de un psicólogo comunitario víctima de las políticas del Estado, o de un estado neoliberal que obliga a los psicólogos comunitarios a ir en contra de los principios de la psicología comunitaria latinoamericana; ya que para Pujal i Llomart (2004) el sujeto no es solo un producto, sino un artífice de dicho proceso de reproducción y transformación de la realidad.

La conclusión para Foucault (1983) sería que el problema político, ético, social y filosófico de nuestros días consiste en enfrentar la subyugación por medio de la sujeción, para lo cual sería necesario concentrarse en impulsar nuevas formas de subjetividad, mediante el rechazo de este tipo de individualidad que se ha impuesto a lo largo del tiempo. Es necesaria la sensación de libertad, de estar eligiendo desempeñarse en una política implementada por alguna ONG, para que la sujeción actúe y, de ese modo, es que el poder del Estado se visibiliza en las prácticas de los psicólogos y psicólogas comunitarias.

Para Foucault (1983), el poder no se ejerce más que sobre sujetos libres: en la medida en que son libres, se enfrentan a un campo de posibilidades donde pueden darse diferentes conductas, reacciones y modos de comportarse; ya que si las determinaciones estuvieran saturadas, no habría relación de poder.

A partir de todo lo anterior, y siguiendo a Flores Osorio (2002), recrear la subjetividad sería transformar al colectivo; en un contexto sociocultural donde descubrir la mismidad significa descubrir a los demás, pues no se puede objetivar la mismidad sin que se manifieste como alteridad y como relación del ser con su otredad. Si es así, y según afirma Sisto Campos (2004), los sujetos no son entidades aisladas o individualizadas, sino más bien, la subjetividad se gesta y desarrolla en las relaciones sociales, ya que desde ahí emergen como sujetos. Para el autor, si el sujeto/a opera en base a significados y queda definido o individualizado desde las relaciones sociales, no puede ser comprendido/a como mónada individual, ya que nuestra propia individualidad emerge de la intersubjetividad.

\section{Voluntariado y psicología comunitaria}

Es posible hipotetizar que el papel de los psicólogos/as comunitarios/as (si se espera la construcción de una subjetividad con énfasis en la participación social y el poder popular) debe, por un lado lidiar con su papel de implementador de las políticas del Estado, y por otro lado, apostar por la construcción de una identidad colectiva. Esto último podría darse en el trabajo colaborativo con pobladores y profesionales de otras disciplinas; pero pensar en una psicología comunitaria en Chile que no se construya desde la implementación de una política pública en particular, sería cada vez más difícil (Alfaro Inzunza \& Zambrano Constanzo, 2009; Asún \& Unger, 2007).

Lo anterior inevitablemente pone en tensión el lugar en el cual se puede desarrollar una psicología comunitaria transformadora, transitando entre lo que Garcés Montoya (2010) diferencia como organizaciones (aquellas con vínculos ideológicos y financieros con instituciones de alta trayectoria de perfiles religioso, político o social) y colectivos (esto es, aquellos impulsados por las comunidades, en respuesta a necesidades o desafíos a la autoridad y a las instituciones). Pero para 
desarrollar un trabajo crítico en colectivos, es necesaria una individualización de la psicología comunitaria, pero esta vez en el sentido en que lo desarrolla Yopo (2013), para quien este término:

sería un proceso que apelaría a mayores niveles de responsabilización de los individuos, que se desarrollaría en contextos de posibilidad heterogéneos y que se constituiría mucho más como mandato que como alternativa libremente elegida. En el proceso de autoproducción los individuos deberían enfrentar el destino de tener que construir su identidad asumiendo la responsabilidad y los costos de dicha tarea (p. 5).

En este sentido, una propuesta colectiva que se reconstruye deberá enfrentar el desafío de la autogestión, en una lógica opuesta a la de una organización formal, tratando de actuar desde una perspectiva diferente a la implementación de políticas del Estado. Este desafío, a su vez, podría generar tensiones, tanto en las relaciones sociales como en la estructura, las que no se encuentran documentadas en estudios previos.

Tal como señalan diversos autores (Alfaro Inzunza, 2007; Alfaro Inzunza \& Zambrano Constanzo, 2009; Asún \& Unger, 2007; Berroeta Torres et al., 2012), si las ONG han debido representar a las políticas del Estado para sobrevivir y obtener recursos, entonces dejan de ser el espacio privilegiado para el desarrollo de una psicología comunitaria con una identidad y subjetividad transformadora, en los términos de Montero (2003, 2008).

Para autores como Fouce Fernández (2009), las ONG se han convertido en la cara comunitaria del neoliberalismo, completando el trabajo destructivo del mismo en proyectos locales. Es entonces cuando aparece la posibilidad de intervenir, desde lo que en países como Argentina ha sido denominado organizaciones del tercer sector (Forni \& Leite, 2006). Si bien este tipo de organizaciones puede incluir a ciertas $\mathrm{ONG}$, en general, se trata de un término que define a "organizaciones que operan por fuera del aparato estatal, que no distribuyen beneficios y a los que los ciudadanos son libres de asociarse o no en la persecución de objetivos comunes" (Forni \& Leite, 2006, p. 219).

En Chile las organizaciones de base como juntas de vecinos y centros comunitarios, estarían cercanas a la definición de Forni y Leite (2006) como organizaciones del tercer sector, destacando sobre todo, por el ejercicio de prácticas de voluntariado para su subsistencia y lógicas de autogestión para poder financiarse.

Sin embargo, es necesario destacar que la lógica del voluntariado no siempre responde a políticas de autogestión y organización de base territorial. Para Salazar y Jaime (2009), en los últimos años, a raíz de una serie de reformas que tenían como objetivo acercar a las personas a las organizaciones, los gobiernos han visto la importancia de integrar a la sociedad civil en la gestión de las políticas públicas, aspecto que hace necesario el fortalecimiento de las organizaciones y el establecimiento de políticas para fomentar el voluntariado.

Entonces, el voluntariado y la promoción de la autogestión en sí mismas tampoco garantizan una praxis transformadora para la psicología comunitaria, necesitando de algo más para volver a conectar a esta disciplina con la interpelación que a inicios de los 80 hacía Montero (1984). En aquel momento la psicología comunitaria debía presentarse como una vía de interacción, 
generadora de tecnología social, cuyo objetivo fuera lograr la autogestión para que los individuos produzcan y controlen los cambios en su ambiente inmediato.

Una praxis transformadora de la psicología comunitaria, podría verse fortalecida por prácticas de voluntariado, siempre que estas se sitúen desde lo que Quintal de Freitas (1994, citado en Castillo \& Winkler, 2010) denominan como psicología de la comunidad (el psicólogo como activista político) y psicología con la comunidad (el psicólogo como catalizador de procesos sociales relevantes para y desde la comunidad).

Además, es necesario construir una mirada crítica de los sentidos del voluntariado. Para Fouce Fernández (2009), el voluntariado y las organizaciones de voluntariado "serían funcionales para el sistema, útiles para responder a algunos retos del mismo" (p. 180). Desde acá, los voluntarios pueden ejecutar una acción poco reflexiva, individual e individualista, actuando como frenos ante posibles cambios sociales o sociopolíticos.

Para Fouce Fernández (2009), el mundo neoliberal necesita de un cuerpo de voluntarios que responda a las inevitables consecuencias negativas, en forma de desigualdades, que genera el capitalismo globalizado. Además, el voluntariado puede actuar como un sector complementario de la acción estatal pública, contribuyendo a la precarización de salarios y condiciones laborales en el ámbito de los servicios de intervención social. Entonces, ¿es posible que el voluntariado se convierta en una alternativa para la psicología comunitaria? Para el autor solo sería posible en el marco de un voluntariado que:

pasa a ser concebido dentro del arco de los movimientos sociales, de los movi- mientos de resistencia; [el voluntariado] pasa a tener, por tanto, una inexcusable dimensión política y crítica, dimensión de cambio social, de reivindicación, de acción colectiva y generalizadora, de lucha por los derechos sociales y por el cambio de estructuras desde la acción concreta (p. 184).

Para Fouce Fernández (2009), esta forma de voluntariado, posible de darse al interior de organizaciones del tercer sector con un énfasis colectivo y no organizacional, solo puede ser verdaderamente crítica si se convierte en una actividad no extraordinaria, instalándose en la vida diaria de las personas, como si se tratara de "estilos de vida solidarios". Esto implicaría "nuestra propia transformación personal y de nuestro estilo de vida o nuestras elecciones" (p. 184), cuestionando entonces la subjetividad del psicólogo y psicóloga comunitarios.

Es a partir de todo lo anterior, que surge la pregunta por las posibilidades de producción concretas de una realidad con estas características, volviéndose relevante investigar algún caso de un centro organizativo de base creado con posterioridad a la Dictadura, en el que se evidencie alguna subjetividad construida en una experiencia de voluntariado o cercana a este, en el marco de la psicología comunitaria. Este artículo muestra los resultados de un estudio de caso, cuyo objetivo fue describir las tensiones de una organización colectiva que se independiza de una $\mathrm{ONG}$, relacionándolas con la subjetividad de psicólogo comunitario que se construye en tal contexto.

\section{El contexto y el caso}

El centro comunitario de este estudio - cuyo nombre y sector geográfico no será mencionado, debido a la solicitud de confidencialidad de sus miembros- no posee 
personalidad jurídica y consiste en un proyecto de carácter comunitario. Este busca desarrollar diversas iniciativas de trabajo colectivo para mejorar la calidad de vida de los pobladores del sector, en una comuna de la Quinta Región de Chile, con la orientación de establecer redes con otras experiencias y territorios que busquen sentidos similares.

El equipo se conoció y constituyó originalmente en el año 2008, trabajando en una ONG que llevaba años en el sector; la cual, por dificultades de gestión, decidió en 2010 cambiar la administración, desde la lógica de un directorio acotado y profesionalizado, a una asamblea general de socios y trabajadores participantes activos de la organización.

De este modo, si bien se respetó la lógica de contar siempre con un director por cada proyecto que la ONG postulaba y se adjudicaba, la administración general pasó, en la práctica, a ser una asamblea constituida con los miembros activos pertenecientes o no en el directorio de la ONG. Por miembros activos, se entiende a los profesionales que en el momento cumplían funciones asociadas a los proyectos, con una carga igual o superior a media jornada laboral (psicólogos, trabajadores sociales y una ingeniera ambiental), así como también a monitoras sin profesión, quienes participaban en proyectos y en el programa de generación de empleos del municipio de la comuna.

Durante los tres años siguientes, la asamblea se reunió periódicamente cada 15 días y por alrededor de dos horas, con el fin de abordar aspectos administrativos y de gestión de proyectos. Este proceso pretendía construir una orgánica basada en lógicas horizontales de toma de decisiones, las cuales fueron tensionando el funcionamiento más vertical que tenían algunos socios fundadores de la ONG, quienes luego del primer año de funcionamiento de la asamblea, cuestionaron su efectividad, argumentando la disminución de proyectos adjudicados por la ONG desde el funcionamiento de esta figura (disminuyeron en un $20 \%$ al primer año y en un $30 \%$ al segundo año).

De los socios fundadores, solo una persona asistía a las reuniones de la asamblea y otra se mantenía informada de las decisiones de la misma. El resto de los socios (cinco) se mantenía al margen de la ONG, dedicándose a otros campos laborales.

A medida que avanzaba el tiempo, el número de proyectos adjudicados fue disminuyendo y la naturaleza de estos cambió: de proyectos de investigación y memoria local, la ONG pasó a adjudicarse proyectos de innovación y emprendimiento. Esto generó el retiro de algunos miembros de la asamblea y la molestia de los socios fundadores.

En el año 2013 esta molestia fue expresada de forma verbal y luego por escrito, lo que llevó a los socios fundadores a informar a la asamblea de su término, con el fin de recuperar la administración de la ONG. Este proceso generó un quiebre entre los miembros de la organización, quienes comenzaron a responsabilizarse de forma cruzada por los cambios. Ante esta tensión, un grupo de ocho miembros, quienes eran los participantes activos de la asamblea, se desvincularon de la ONG para fundar un centro comunitario en el mismo territorio.

Entre el grupo de personas que conforman dicho centro, se encontraban monitoras de estimulación oportuna que vivían en el sector, una ingeniera ambiental y varios psicólogos y psicólogas. En el proceso de 
fundación de este centro, el grupo hizo una alianza con una naciente ONG de Valparaíso, con el fin de contar con la personalidad jurídica de la misma y así postular a licitaciones y proyectos públicos.

Durante los primeros siete meses, el centro comunitario funcionó físicamente en una casa pequeña arrendada con aportes personales de sus miembros. Luego de ese período, consiguieron una oficina en una sede de la Junta de vecinos del sector, alternando la personalidad jurídica de esta última y de la ONG de Valparaíso para postular a proyectos.

Con excepción de dos monitoras, que recibieron una remuneración de alrededor de ciento diez dólares mensuales, por ser parte del Programa de Generación de Empleo del Servicio Nacional de Capacitación y Empleo, el resto de los integrantes comprometió un número de horas de trabajo voluntario con tareas específicas.

\section{Metodología}

\section{Diseño}

El presente estudio corresponde a una investigación cualitativa (Rodríguez Gómez, Gil Flores, \& García Jiménez, 1996) y posee un diseño de estudio de caso único (Stake, 1998). La decisión del método se basa en los aportes de Rodríguez et al. (1996), para quienes los diseños de caso único centran su análisis en un proceso singular, debido a su potencial contribución al conocimiento, así como a su carácter extremo, lo cual se verifica aquí.

El procedimiento de producción de información tuvo características etnográficas, específicamente, autoetnográficas (Denzin \& Lincoln, 2002; Duncan, 2004; Ellis \& Bochner, 2000); ya que hay presentes ele- mentos de experiencia personal en la investigación (Clandinin \& Connelly, 1994).

Para Scribano y De Sena (2009), la autoetnografía es un modo de etnografía que consiste en aprovechar las experiencias afectivas y cognitivas de quien quiere elaborar conocimiento acerca de un aspecto de la realidad, basándose en la propia participación en este, coincidiendo con las definiciones de Denzin y Lincoln (2002). En este caso, dos de los autores de ese estudio participan activamente del centro comunitario.

Técnicas de producción de información y participantes

Se llevaron a cabo observaciones participantes transcritas como notas de campo (Pérez Serrano, 1994). Así mismo, se utilizaron las actas de las asambleas como registro de apoyo a las observaciones. El procedimiento de producción de información consistió en realizar observaciones participantes persistentes (Ruiz-Olabuénaga, 1996) por un periodo de seis meses (julio a diciembre del año 2014), registrando aquellas que eran consideradas como hitos para el propio equipo observador.

Las primeras observaciones generaron información relevante que constituyó la pauta de las primeras entrevistas, conformadas por preguntas acerca de la experiencia de trabajo en la ONG de la cual se habían retirado, así como también respecto de las proyecciones personales y laborales en el naciente centro comunitario.

Algunas entrevistas tuvieron carácter semiestructurado, mientras que otras se desarrollaron como entrevistas activo-reflexivas (Denzin, 2001), las que constituyen una interacción en la cual los participantes son asumidos como sujetos que interactúan 
abiertamente y la entrevista es considerada como una forma de acción social.

A medida que se avanzó en este proceso, se fue también progresando de entrevistas semiestructuradas a entrevistas activoreflexivas. Los últimos diálogos preguntaban acerca de los significados construidos respecto de la experiencia actual en el centro comunitario, con énfasis en el desarrollo disciplinar, así como en las relaciones que se establecieron con la comunidad de vecinos.

Las entrevistas fueron realizadas de forma alternada por los investigadores autores de este artículo. Las observaciones se desarrollaron en espacios informales (acompañándolos a buscar el espacio de arriendo para el centro comunitario, yendo a conversar con la Junta de vecinos para instalarse en sus dependencias, entre otras instancias), pero las entrevistas fueron realizadas en un espacio físico adecuado (oficina del centro comunitario) y sin interrupciones, con el fin de profundizar en torno a los diferentes significados.

Como parte de la producción de información, se hizo un grupo focal (Canales Cerón, 2006) con el fin de describir las experiencias colectivas y significados compartidos. En este se identificaron colectivamente ciertos hitos que los participantes consideraron relevantes para describir su historia como centro comunitario. Las observaciones fueron realizadas por los investigadores que, además, formaban parte del centro comunitario. En la tabla 1 se muestra el resumen de las observaciones realizadas y en la tabla 2 , las características de los/las entrevistados/as.

\section{Tabla 1}

\section{Resumen de observaciones registradas}

\begin{tabular}{|c|c|}
\hline Técnica & Personas involucradas y momento observado \\
\hline Observaciones iniciales & $\begin{array}{l}\text { 1. Reunión de miembros que se desvincularon de la } \\
\text { ONG de origen, en esta se decide la desvinculación y } \\
\text { formación de un centro comunitario. } 19 \text { de junio, } 2014 \text {. } \\
\text { 2. Búsqueda de un espacio físico por } 5 \text { miembros del } \\
\text { Centro Comunitario. Implicó visitas a casas en arriendo } \\
\text { en el sector, así como conversaciones informales res- } \\
\text { pecto de las características que debía cumplir la casa } \\
\text { que eligieran. } 26 \text { de junio, } 2014 \text {. }\end{array}$ \\
\hline Observaciones de asambleas & $\begin{array}{l}\text { Asambleas de miembros del Centro Comunitario: asis- } \\
\text { ten todos sus miembros: psicólogos/as, ingeniera y mo- } \\
\text { nitora. A algunas asambleas asisten practicantes. } 3 \text { de } \\
\text { julio, } 23 \text { de julio, } 13 \text { septiembre, } 24 \text { de septiembre, } 18 \\
\text { octubre, } 12 \text { noviembre y } 11 \text { diciembre; } 2014 \text {. }\end{array}$ \\
\hline \multirow[t]{2}{*}{$\begin{array}{l}\text { Otras observaciones } \\
\text { relevantes }\end{array}$} & $\begin{array}{l}\text { 1. Actividad de Fiestas Patrias: consistió en un conjunto } \\
\text { de actividades dirigidas a los vecinos del sector (venta } \\
\text { de anticuchos, pinta caritas, entre otros). } 19 \text { de septiem- } \\
\text { bre, } 2014 \text {. }\end{array}$ \\
\hline & $\begin{array}{l}\text { 2. Jornada de reflexión realizada por un grupo de estu- } \\
\text { diantes en práctica temprana de psicología. } 24 \text { de sep- } \\
\text { tiembre, } 2014 \text {. }\end{array}$ \\
\hline
\end{tabular}


Tabla 2

Características de los/as entrevistados/as

\begin{tabular}{|c|c|c|c|c|}
\hline $\begin{array}{l}\text { Técnica de } \\
\text { producción de } \\
\text { información }\end{array}$ & Participante & Sexo & Edad & $\begin{array}{l}\text { Año de } \\
\text { incorpo- } \\
\text { ración al } \\
\text { equipo }\end{array}$ \\
\hline \multirow{9}{*}{$\begin{array}{l}\text { Entrevista } \\
\text { activa- } \\
\text { reflexiva }\end{array}$} & Ingeniera ambiental & Mujer & 30 a 39 & 2008 \\
\hline & Psicólogo clínico & Hombre & 30 a 39 & 2013 \\
\hline & Monitora social & Mujer & 30 a 39 & 2007 \\
\hline & Practicante psicología & Mujer & 40 a 49 & 2013 \\
\hline & Psicóloga 1, ex practicante & Mujer & 20 a 29 & 2012 \\
\hline & Psicóloga 2, ex practicante & Mujer & 20 a 29 & 2012 \\
\hline & Psicólogo 3, ex practicante & Hombre & 20 a 29 & 2014 \\
\hline & $\begin{array}{l}\text { Psicólogo, coordinador de prácticas } \\
\text { profesionales }\end{array}$ & Hombre & 30 a 39 & 2008 \\
\hline & Psicóloga & Mujer & 30 a 39 & 2012 \\
\hline Grupo focal & $\begin{array}{l}\text { Psicólogo clínico, psicólogo coordi- } \\
\text { nador de prácticas, psicóloga, estu- } \\
\text { diante en práctica de psicología, } \\
\text { monitora social } 1 \text {, monitora social } 2 \text {, } \\
\text { ingeniera ambiental }\end{array}$ & & & \\
\hline
\end{tabular}

Procedimiento y aspectos éticos

En la segunda asamblea se explicó la intención de llevar a cabo un proceso sistemático de producción y análisis de información desde una perspectiva situada (Montenegro, 2001), considerando que dos investigadores psicólogos formaban parte de la organización. En este sentido, cada investigador llevaba un cuaderno de campo con notas de los elementos centrales de la experiencia de desvinculación de la ONG de origen, los que decidieron socializar con el resto de los miembros y formalizar un proceso científico de indagación.

Luego de un mes, se llevó a cabo el proceso de selección de participantes del estudio (Salamanca Castro \& MartínCrespo Blanco, 2007) para realizar las primeras entrevistas, lo que rápidamente avanzó a un muestreo intencionado, con el fin de preguntar en las entrevistas los aspectos centrales de las observaciones realizadas. Cada entrevista fue grabada y transcrita, solicitando los respectivos consentimientos informados. Con el fin de resguardar la confidencialidad de la información, los nombres de los participantes han sido cambiados en este artículo.

\section{Técnicas de análisis de información}

Para analizar la información producida en este estudio, se llevó a cabo un análisis de contenido categorial temático, siguiendo las recomendaciones de Vázquez (1994), desarrollando tres etapas consecutivas y recursivas: preanálisis, codificación y categorización. Los datos fueron analizados en el programa Atlas ti versión 7.0 (Educativa Licencia Multiusuario 5 Unidades, 2015). Los criterios de rigor científico contemplaron la credibilidad (triangulación de méto- 
dos, datos y fuentes) y la dependencia, a través de una auditoría de confirmación (Ruiz-Olabuénaga, 1996) solicitándoles a los miembros de la ONG que comprobaran la correspondencia entre los datos y las interpretaciones realizadas. Las categorías buscaron identificar los temas centrales que se presentaban en tensión, dando cuenta, además, de las formas en que se resolvían.

\section{Resultados}

A continuación, se presentan dos categorías que expresan las dos tensiones más relevantes en este centro comunitario. Por un lado, la primera categoría denominada "El papel de la psicología en el centro comunitario", muestra cómo es la psicología como disciplina, la que se tensiona identitariamente en este centro comunitario, transitando entre diversas formas de "ser psicólogos/as".

La segunda categoría, denominada "Sobre la función social del centro y el voluntariado como mecanismo para esa función" se vincula con el impacto psicosocial de las acciones en el centro, el cual es analizado como una influencia política que utiliza estrategias de voluntariado para poder cumplir esta función política.

\section{Categoría 1. Tensiones disciplinares: El} papel de la psicología en el centro comunitario

El centro comunitario no tiene personalidad jurídica, como se mencionó antes, por lo que -en rigor- no puede postular a proyectos concursables. Ello es complejo, en términos de la identidad del propio centro, ya que hasta ese momento el equipo de personas se reunía a través de una identidad laboral, con el fin de trabajar en proyectos sociales y comunitarios. Así, surgen temores asociados a la imposibilidad de generar recursos que permitan, no solo la subsistencia del centro, sino que potencien la permanencia de sus miembros en él.

La necesidad de ganar proyectos concursables con fondos que más allá de los recursos impliquen un aliciente al ánimo del equipo (Psicólogo, coordinador de prácticas profesionales; segmento de entrevista).

A mí lo que me da miedo es que al final, nos vamos a terminar convirtiendo en el Hogar de Cristo, o mejor dicho, me da miedo que nos convirtamos en eso, porque esto debiese ser más político, autogestionado si quieres, pero no solo voluntariado, que me cuesta verlo como una inversión a largo plazo (Psicóloga, segmento de grupo focal).

La metáfora respecto del Hogar de Cristo ${ }^{1}$ refiere a una condición de asistencialismo que es resistida por el equipo. Si bien, por un lado, se concluye que la obtención de recursos económicos sería un motivador para que las personas se mantengan en el proyecto, los significados no solo se vinculan con que exista una fuente de ingresos, sino con la posibilidad de darle continuidad a una propuesta emergente. En este sentido, se cuestiona el voluntariado como un elemento de potenciación del proyecto colectivo.

Yo entiendo lo de los practicantes y que sean voluntarios. Lo encuentro choro, pero también cuando es voluntario, el tiempo se hace escaso, porque igual tenís que ver cómo ganar algo de plata si no es pa' tu sueldo a fin de mes, que sea al menos pa' los materia-

1 El Hogar de Cristo es una institución chilena de beneficencia pública fundada por el sacerdote jesuita Alberto Hurtado. 
les. Igual hay hartos materiales, pero no sé... yo creo que es sentir que se valora poco el trabajo si no hay nadanada de sueldo... (Psicólogo 3, ex practicante; segmento de entrevista).

Yo a veces necesito como el piso que tenía la ONG de la que nos fuimos, porque igual era conocida... no sé, mira, ponte si hago un curso acá llegan unas 10 personas, salva pa'l arriendo, pero allá me podía hacer el sueldo de un mes en un curso de 4 días. Yo he pensado que más que armar una personalidad jurídica, lo mejor es asumirnos como parte de [nombra la ONG] que actualmente permite que postulen proyectos con su personalidad jurídica, como una sucursal, o el centro comunitario de la ONG, por ejemplo (Psicóloga, segmento de grupo focal).

Por un lado, existe una asociación entre remuneraciones y valoración de la profesión, lo que implicaría que la identidad de trabajo se vincula con el valor económico del mismo. Por otro, se evidencia la necesidad de poseer una estructura delimitada formalmente. Esto podría estar dando cuenta de una necesidad de sujeción mediante el control y la dependencia, lo que se evidencia en la necesidad de definir al colectivo como una organización para, desde allí, establecer las proyecciones y el posicionamiento como centro comunitario. Esto implicaría un sentido identitario: en la medida en que haya un referente institucional con el cual identificarse, es posible definir la propia labor e, incluso, sería viable proyectar acciones profesionales.

Es en este punto donde comienzan a converger diferentes identidades colectivas. Para algunos miembros, este centro constituye un sueño colectivo y se destaca el valor del proyecto común, aunque queda establecido que no hay claridad respecto de ciertos elementos de funcionamiento interno, así como de algunas metas que le dan sentido a la organización. Desde esta perspectiva, habría ciertos componentes de idealización de la función del centro, de la cual la psicología como disciplina aparece con un rol importante, ya que otras disciplinas no encuentran un lugar identitario en esta organización.

Este centro es un sueño (Monitora social, segmento de entrevista).

Compañeros, es decir, partes de un mismo equipo, esto en el entendido que es sacar un proyecto adelante, desde distintas ópticas, aunque con los mismos objetivos (Estudiante en práctica de psicología, segmento de grupo focal).

Tener un diagnóstico organizacional de nuestra propia forma de funcionar yo creo que nos va a servir pa' entendernos un poco más, para tomar decisiones más informados de cómo funcionamos o cómo podemos llegar a funcionar mejor (Psicóloga, segmento de grupo focal).

Y es que yo soy psicóloga po', y me gusta serlo, yo soy repsicóloga [ríe] y que sea educacional o con cosas comunitarias no me hace menos psicóloga, entonces mi rol es hacerme la pregunta sobre cómo, desde mi ser psicóloga y no vecina, puedo aportar acá. $\mathrm{Pa}$ ' mí por eso fue importante que con el Juan nos ganáramos el proyecto de primer apoyo psicológico, porque ahí tú tienes la psicología al servicio de la acción comunitaria, porque son pobladores los que aprenden a hacer primer apoyo y es una forma de democratizar la disciplina, de que no sea elitista, de unos pocos. Porque ahí yo 
no corto con ni uno, todo se gasta en las capacitaciones, pero con eso me basta, con la plata pa'l café y con sentir que esto sirve pa' algo y pa' alguien (Psicóloga, segmento de entrevista).

Actualmente, en la etapa de formación de este centro mis intereses personales se ven muy poco reflejados (Ingeniera ambiental, segmento de entrevista).

En el centro comunitario se han llevado a cabo dos proyectos con fondos externos, uno de los cuales implicó un trabajo de capacitación de primer apoyo psicológico en contextos de crisis y desastres a pobladores. Esta experiencia, desde la perspectiva de algunos miembros, sitúa a la psicología, en términos disciplinarios, como un lugar de acción en la comunidad; impactando, además, en lo que es interpretado como la democratización del conocimiento psicológico y empoderando a los actores sociales en materias disciplinares.

Sin embargo, el papel de la psicología no está dado tan solo por la socialización o democratización de los saberes disciplinares. Para algunos miembros, la psicología se va conformando como una disciplina encargada de canalizar la reivindicación social vía participación solidaria, con un énfasis en la autogestión y organización territorial.

Con este fin, paulatinamente se ha ido construyendo un discurso de identidad local en relación con el sector territorial en el que estos intervienen. No obstante, las posiciones desde las cuales se sitúan para el conocimiento de este territorio son diferentes dependiendo del sujeto que enuncie tal posición. Por ejemplo, los psicólogos y psicólogas identifican las necesidades de los pobladores, mientras que las monitoras se identifican con las necesidades.

En este sentido, se genera una distancia entre el rol del psicólogo como mediador -que acompaña a los pobladores en un tránsito desde conciencias ingenuas o mágicas hacia conciencias críticas- y de quienes protagonizan sus propios cambios. Ninguno/a de los/las psicólogos/as vive en el sector, generando, entonces, una identificación que no es de pertenencia, a diferencia de las monitoras, que sí viven en el sector.

Lo revelador es que soy de aquí [nombra al sector], conozco lo que se necesita en mi comunidad y por ser de aquí los proyectos pensados se tratan de las problemáticas de aquí (Monitora social, segmento de grupo focal).

Salimos a caminar y conocer los nombres de las calles. Esto era un paso necesario, ya que era la primera vez que yo venía (Estudiante en práctica de psicología, segmento de grupo focal).

Yo me acuerdo cuando Miguel me agarró y me dijo "ya cabrito, a caminar" y empezamos a hacer puerta a puerta y me moría de miedo, porque yo no conocía el lugar, no es mi barrio, ni entiendo así de fácil las dinámicas poblacionales. $\mathrm{Mi}$ supervisor fue de verdad supervisor y asesor de práctica (Psicólogo 3, ex practicante; segmento de entrevista).

Yo siempre he pensado en venirme a vivir acá. Lo hemos conversado harto, porque igual me pregunto por qué no estoy trabajando en la Junta de vecinos de al lado de mi casa, que es mi sector, etc. Pero como mi casa no es mi casa, si po', sí me podría venir para 
acá. Igual me pregunto si quiero, si quiero que mi hija crezca acá, y sí po', con hartas contradicciones ahí (Psicóloga, segmento de entrevista).

Una de las principales tensiones que se dejan ver en el papel de los psicólogos, es la relación identitaria y de pertenencia con el sector intervenido, el cual se convierte en un sector ajeno, que es necesario conocer y comprender. Frente a la pregunta de las motivaciones que están en la base para actuar en un contexto geográfico diferente al de procedencia, se van construyendo significados diversos. Para algunos, las acciones implementadas parecen impuestas, externas a las lógicas barriales; mientras que otros relevan el papel del aprendizaje con la comunidad.

Yo a veces me pregunto si no estaremos presionando procesos que no son propios de lo comunitario, o mejor dicho, de la vida comunitaria. Tú ves lo difícil que es juntar a la gente, lo vimos con el proyecto Fosis, y los tiempos de las comunidades no son los tiempos de los proyectos (Psicólogo 3 , ex practicante; segmento de entrevista).

No espero impactar. Solo vengo a aprender y a compartir lo aprendido con quienes ya son sabias mujeres (Estudiante en práctica de psicología, segmento de grupo focal).

Los sentidos durante este corto periodo de trabajo en aquel ámbito giran en torno a la realización de un trabajo territorial, en donde existe la necesidad de fortalecer los espacios de encuentro entre los actores y actrices que están conviviendo en el territorio (Psicóloga 1, ex practicante; segmento de entrevista).
Al tratar de comprender qué lleva a los profesionales a trabajar en este sector, aparecen ciertos principios: la participación, el consenso, la articulación y organización del sector territorial. De este modo, existe el diagnóstico de que los vecinos y vecinas se sienten aislados $\mathrm{y}$ aisladas, y que, pese a tener el entusiasmo de participar de actividades de organización local, no lo hacen. De este modo, el trabajo de la psicología aparece como una invitación a la promoción de la organización colectiva, en contextos de atomización social.

Yo destaco que en el Fosis la gente se juntara a hacer la placita, si fue lo que iniciaron los practicantes, pero ahora ya está en las manos de los vecinos. Fue bonito ver que gente que se ve todos los días pero que de verdad no se ve, ahora se viera. Sé que fueron pocos, pero no importa. Estamos en una sociedad tan individualista, que la gente no vive en el barrio, sino que transita y que se logren reunir, que dejen de ser átomos en la pobla es bonito, eso sí es participar y consensuar (Psicóloga, segmento de entrevista).

Juan: Ya po' pero qué vamos a hacer al final.

Flor: ¡Una peña! [risas generales].

Clara: ¿Cómo una peña? [ríe] Tiene que ser en serio, algo piola que podamos hacer, pero que la gente nos conozca. Algo que permita que sepan quiénes somos, pero sobre todo que quieran venir a estar juntos.

Sergio: Hagámoslo en la calle, si total acá no pasa mucho. Y ponemos tarros, pa' que tiren pelota y tiramos la parrilla...

Flor: Sí, así los vecinos acá empiezan a hablar, porque no es como en el paradero [nombra un sector] acá no se hablan. 
Juan: Y ¿cómo saben? ¡Ahí empieza la articulación social, cabros! [risas generales]

(Observaciones de asamblea).

Categoría 2. Tensiones políticas: Sobre la función social del centro y el voluntariado como mecanismo para esa función

El énfasis en la articulación colectiva como meta de las acciones del centro comunitario, se presenta como parte de un proyecto político mayor, dando cuenta del quehacer de la psicología como una actividad política en sí misma, así como una disciplina catalizadora de procesos sociales relevantes para y desde la comunidad. Esto se materializa en las preocupaciones acerca de la calidad de vida y de la necesidad de que la organización colectiva se base en permanentes procesos de reflexión crítica hacia una construcción transformadora de las comunidades más pobres y marginadas.

Mi interés es poder construir iniciativas con los pobladores que permitan, progresivamente, la mejora de la calidad de vida del sector, además de generar procesos de reflexión crítica acerca de su propia situación y los factores estructurales y de comunidad que facilitan que puedan sentirse excluidos u oprimidos. Creo que con el desarrollo de estos proyectos es posible ir avanzando en estos aspectos desde la práctica y encender la mecha de la pradera transformativa. El trabajo comunitario, para mí, no puede separarse de una imagen de sociedad e ideal político que uno puede tener explícita o implícitamente (Psicólogo, coordinador de prácticas profesionales; segmento de entrevista).

Este centro comunitario no es cualquier centro. No es un centro asisten- cial, ni un centro deportivo. Si al final, es raro porque no tenemos identidad. Pero no la tenemos porque no nos interesa tenerla, porque la apuesta es política, lo que interesa es colaborar con procesos de toma de conciencia que articulen y empoderen a quienes sí son protagonistas de este espacio (Psicólogo 3 , ex practicante; segmento de entrevista).

Ya, yo sé que es extremo lo del Hogar de Cristo, pero iy si no es un centro asistencial qué es? No es un lugar de trabajo, no remunerado ¿cierto? Acá no venimos a ganar plata [ríe] pero ¿entonces a qué venimos? [silencio]. Venimos porque esta es una organización política, con un frente de batalla definido. Estamos tratando de recuperar la inercia y el individualismo en que nos dejó la Dictadura, estamos tratando de recuperarnos a nosotros mismos, y por eso no se cobra, eso se hace con la voluntad de un pueblo (...) pero lo que preguntas es que... por eso esto es voluntariado, pero claro, no como el Hogar de Cristo, si exagero, es un voluntariado más combativo, diría yo (Psicóloga, segmento de entrevista).

Una forma que este grupo ha encontrado de resolver las tensiones identitarias, en términos disciplinarios y políticos, ha sido la organización colectiva a través de ciertas prácticas que implican una subjetividad de voluntariado, a la vez que buscan ciertos soportes institucionales para hacer efectivas las intervenciones. De este modo, los aportes del grupo son concebidos como personales, y las horas de tiempo de voluntariado han sido significadas como una forma de "aportarse" a uno mismo, más que de aportar horas. De este modo, sin querer, han quedado jerarquizados, ya que quien aporte con más horas 
de voluntariado, será reconocido como aquel con mayor ejercicio de autoridad.

Así, cada uno de los que están en el centro reconoce explícitamente un rol, quedando sujetos a la propia identidad. Los discursos poseen la carga simbólica del bien y el mal, en el simbolismo del amor. Las personas de este centro necesitan reunir una cantidad de dinero mensual para el pago de cuentas, pero algunos utilizan la metáfora de "vivir de amor" para presentarse, como si el aporte personal fuese una muestra de amor.

Entrevistador: ¿Pero se sostiene de algo?

Psicóloga: De nuestros aportes por el momento.

Psicólogo, coordinador de prácticas profesionales: Se sostiene de amor.

Psicóloga: Vivimos de puro amor [risas] (Segmento de grupo focal).

Cuando yo dije que vivimos de amor, lo que quise decir es que no tenemos plata [ríe]. Es que no la tenemos, o tal vez ahora tenemos un poco más, pero hemos vendido empanadas, hecho sopaipillas, etcetera, y a mí me gustaría que el centro se sostuviera por sí mismo, pero a la vez entiendo que es una apuesta política que hay que mantener (Psicóloga, segmento de entrevista).

Lo que me preguntas de si alguien es como el jefe, es que a veces sí, pero se supone que no. Es que igual el Juan tiene un día fijo en que sube y está con los practicantes, y es como el encargado, y es con quien hablan en la Junta de vecinos, porque él entrega el día completo acá, y yo creo que es el que más comprometido está (Psicólogo 3, ex practicante; segmento de entrevista).
Sin embargo, y aunque el riesgo del voluntariado sea la clasificación y el rango, este conjunto de personas no visualiza otra posibilidad por el momento, ya que han debido buscar trabajo remunerado asociado a otras instituciones, con el fin de solventar sus propias vidas. Esto expresa la actual precarización de este tipo de iniciativas, así como las dificultades para construir un trabajo en psicología comunitaria que no esté de la mano de alguna implementación de política pública, generando un desgaste en los psicólogos que participan de iniciativas como esta.

Venimos de la otra organización y ahora estoy dedicada a trabajar en otro lado, porque un momento de acá sin proyectos, sin digamos entrada económica, por lo tanto, tuve que trabajar en otra cosa no más po' (Monitora social 2, segmento de grupo focal).

Ahora estoy trabajando en un liceo y eso son 20 horas y el resto de las horas trato de dedicarlas acá (Psicólogo, coordinador de prácticas profesionales; segmento de grupo focal).

No es posible tener un sueldo fijo para quienes allí se desempeñan, por eso la necesidad de trabajar en otros lugares y dividir los tiempos para participar en el centro, con harto desgaste para quienes lo llevan a cabo (Psicóloga 2, ex practicante; segmento de entrevista).

Con el fin de hacer frente a esta precarización e implementar acciones comunitarias en un contexto de voluntariado, con poco tiempo y sin recursos, la estrategia diseñada hasta ahora ha sido la de crear vínculos con las universidades de la zona. De tal modo, se cuenta con estudiantes en práctica, hasta ahora todos de psicología, 
que tengan la disponibilidad horaria para llevar a cabo intervenciones de carácter comunitario, bajo la supervisión de uno de los psicólogos que se desempeña en el centro.

Esto podría estar favoreciendo la apertura de ciertas universidades a una comunidad con un colectivo que puede, paulatinamente, ofrecer los apoyos necesarios para combinar la formación de futuros psicólogos y psicólogas, con un enfoque centrado en las necesidades de la comunidad.

Acabo de llegar con mi hija a la placita y está bellísima. No había alcanzado a ver lo que estaban haciendo esta semana, pero se ve preciosa: la gente, toda reunida alrededor del escenario, y viene el doble de ¡Camilo Sesto! Mi hija corre con los otros niños y se pelean por los neumáticos que parecen columpios, la gente le da las gracias a los practicantes y a Juan, es emocionante (Psicóloga, segmento de grupo focal).

La actividad realizada por estudiantes en práctica de nuestro centro ha generado un gran impacto en mis vecinos y pretendo que esto pueda seguir y generar una mejor convivencia entre vecinos y vecinas (Monitora social, segmento de entrevista).

$\mathrm{Si}$ al final nos sostenemos gracias a los practicantes. Obvio que no solo gracias a ellos, pero en gran parte. Y es raro porque ahí nosotros creemos que hay voluntariado, pero la verdad es que no. Voluntariado es lo que nosotros hacemos, ellos están obligados y nosotros sabemos usar esa obligatoriedad como si fuera una alianza (Psicólogo 3, ex practicante; segmento de entrevista).

\section{Discusión}

Para Montero (2008), en la psicología comunitaria se vuelve crucial el "poder y control sobre las circunstancias de vida por parte de las personas que integran las comunidades, así como el cambio social como objeto de esta rama de la psicología" (p. 77). En este sentido, los discursos de todos los actores sociales acá implicados apuntan a ir desarrollando paulatinos procesos de toma de conciencia en las personas con las cuales trabajan, es decir, los pobladores del sector.

Sin embargo, existen diversas tensiones respecto de lo que Montero (2008) desarrolla cuando establece la delimitación de la relación entre psicólogos comunitarios y otros actores sociales. Si bien hay matices entre este grupo de psicólogos que se orientan a establecer una relación de acompañar a quienes, supuestamente, no han logrado el desarrollo de una conciencia crítica hacia mayores niveles de empoderamiento, en el caso estudiado conviven las nociones de psicología en la comunidad y psicología para la comunidad (Castillo \& Winkler, 2010).

La identidad colectiva de los/las psicólogos/as que trabajaban en la ONG en la que se conocieron, se había construido desde la implementación de políticas del Estado (Alfaro Inzunza \& Zambrano Constanzo, 2009; Asún \& Unger, 2007) y ha ido transitando hacia el establecimiento de vínculos más bien colectivos (Garcés Montoya, 2010) que sitúan a la comunidad autogestionada en el centro de su accionar.

Este tránsito genera ciertas tensiones, ya que se comienzan a cuestionar concepciones y prácticas, pero sobre todo, el 
sentido y meta de la propia organización. En un primer momento, era la política pública la que otorgaba un fin claro (Berroeta Torres et al., 2012), asociado a los diferentes proyectos concursados, mientras que ahora es la autogestión la que opera como motor de las acciones cotidianas (Salazar \& Jaime, 2009).

Una consecuencia de esto es la convergencia de diversas identidades colectivas. Para algunos miembros, este centro constituye un sueño colectivo y se destaca el valor del proyecto común; aunque queda establecido que no hay claridad respecto de ciertos elementos de funcionamiento interno, así como de algunas metas que le dan sentido a la organización. Desde esta perspectiva, habría ciertos componentes de idealización, asociados al rol político del centro (Asún \& Zúñiga, 2013), expresado como profundización democrática y ampliación de la participación ciudadana (Fernández, 2013).

Sin embargo, existen otros miembros no psicólogos/as que no encuentran un lugar en este "sueño colectivo". Lo anterior se fortalece con el hecho de que los pocos proyectos que han implementado hasta ahora parecieran tener un énfasis disciplinar.

A pesar del necesario cuestionamiento respecto de las relaciones de poder y autoridad establecidas entre los miembros de este centro comunitario, y entre ellos y el resto de la comunidad, resulta interesante que los cuestionamientos -no solo de los/las psicólogos/as que se desempeñan en este centro, sino de todos- se vinculen con la meta de generar la consciencia de que es posible cambiar las prácticas sociales que producen la situación injusta; a través de la acción colectiva y la movilización (Paredes, 2013), apostando por la promoción de mayor agenciamiento.

Sin embargo, tal como se planteó anteriormente, este cambio en las prácticas sociales introduce diferentes tensiones respecto del sentido y finalidad de las intervenciones, considerando la dependencia de financiamiento de las instituciones para subsistir (Berroeta Torres et al., 2012).

Este grupo de personas ha encontrado en el voluntariado crítico (Fouce Fernández, 2009) y en la alianza con las universidades, una posibilidad de acción para implementar estrategias transformadoras; aunque esto signifique, a veces, asumir que existirá una relación de autoridad desde quien detente mayor número de horas de voluntariado.

En síntesis, es de esperar que convivan diferentes lógicas en el accionar de los/las psicólogos/as comunitarios/as, toda vez que las políticas públicas tienden a una mayor institucionalización del quehacer (Asún \& Unger, 2007) y la subjetividad se va construyendo a partir de sujetos/as atados/as a la propia identidad creada, no solo por una conciencia de sí, sino por un marco posible de acción.

\section{Referencias}

Alfaro Inzunza, J. (2007). Políticas sociales como condición de posibilidad para el desarrollo de prácticas en psicología comunitaria. En J. Alfaro Inzunza \& H. Berroeta Torres (Eds.), Trayectoria de la psicología comunitaria en Chile. Prácticas y conceptos (pp. 43-72). Valparaíso, Chile: Universidad de Valparaíso. 
Alfaro Inzunza, J. \& Zambrano Constanzo, A. (2009). Psicología comunitaria y políticas sociales en Chile. Psicologia y Sociedade, 21(2), 275-282.

http://dx.doi.org/10.1590/s0102-71822009000200015

Asún, D. \& Unger, J. (2007). Una visión regional de la institucionalización de la psicología (social) comunitaria en Chile. En A. Zambrano, G. Rozas, F. Magaña, D. Asún, \& R. Pérez-Luco (Eds.). Psicología comunitaria en Chile: evolución, perspectivas y proyecciones (pp. 213-226). Santiago, Chile: RIL Editores.

Asún, R. \& Zúñiga, C. (2013). ¿Por qué se participa? Explicando la protesta social regionalista a partir de dos modelos psicosociales. Psicoperspectivas, 12(2), 38-50.

http://dx.doi.org/10.5027/psicoperspectivas-Vol12-Issue2-fulltext-260

Berroeta Torres, H., Hatibovich Diaz, F., \& Asún Salazar, D. (2012). Psicología comunitaria: prácticas en Valparaíso y visión disciplinar de los académicos nacionales. Polis, 11(31), 335-354.

http://dx.doi.org/10.4067/S0718-65682012000100018

Bustos Titus, L. (1996). Educación popular: lo que va de ayer a hoy. Última Década, 4, 19. Recuperado de

http://www.redalyc.org/articulo.oa?id=19500402

Canales Cerón, M. (2006). Metodologías de investigación social: introducción a los oficios. Santiago, Chile: LOM Ediciones.

Castillo, J. \& Winkler, M. I. (2010). Praxis y ética en psicología comunitaria: representaciones sociales de usuarias y usuarios de programas comunitarios en la Región Metropolitana. Psykhe, 19(1), 31-46.

http://dx.doi.org/10.4067/S0718-22282010000100003

Clandinin, D. J. \& Connelly, F. M. (1994). Personal experience methods. En N. K. Denzin \& Y. S. Lincoln (Eds.), Handbook of qualitative research (pp. 413-427). London, England: SAGE.

Denzin, N. K. (2001). The reflexive interview and a performative social science. Qualitative Research, 1(1), 23-46.

http://dx.doi.org/10.1177/146879410100100102

Denzin, N. K. \& Lincoln, Y. S. (2002). The qualitative inquiry reader. Thousand Oaks, California: SAGE.

Díez Rodríguez, A. (1999). Voluntarios, ONGs y sociedad civil en la reordenación globalizadora. Estudios de Juventud, 45, 93-102. Recuperado de http://www.injuve.es/sites/default/files/Revista45-9.pdf

dos Santos Azevêdo, A. V. (2009). A psicologia social, comunitária e social comunitária: definições dos objetos de estudo. Psicologia Em Foco, 3(2), 64-72. Recuperado de $\mathrm{http} / / /$ periodicos.piodecimo.edu.br/online/index.php/psicologioemfoco/article/view/46/65

Duncan, M. (2004). Autoethnography: Critical appreciation of an emerging art. International Journal of Qualitative Methods, 3(4), 1-14. Recuperado de https://www.ualberta.ca/ iiqm/backissues/3_4/pdf/duncan.pdf 
Educativa Licencia Multiusuario 5 Unidades (2015) Atlas.ti7 (7.5.10) [software computacional]. Berlin: Scientific Software Development GmbH.

Ellis, C. \& Bochner, A. P. (2000). Autoethnography, personal narrative, reflexivity. En N. K. Denzin \& Y. S. Lincoln (Eds.), Handbook of qualitative research (pp. 733-768). Thousand Oaks, California: SAGE.

Fernández, R. (2013). El espacio público en disputa: manifestaciones políticas, ciudad y ciudadanía en el Chile actual. Psicoperspectivas, 12(2), 28-37.

http://dx.doi.org/10.5027/psicoperspectivas-Vol12-Issue2-fulltext-278

Flores Osorio, J. (2002). Epistemología de la subjetividad ¿mesoamericana? En I. Pipper (Comp.), Políticas, sujetos y resistencias: debates y críticas en psicología social (pp. 267-278). Santiago, Chile: Arcis.

Forni, P. \& Leite, L. V. (2006). El desarrollo y legitimación de las organizaciones del tercer sector en la Argentina. Hacia la definición de un isomorfismo periférico. Sociologías, 16, 216-249. http://dx.doi.org/10.1590/S1517-45222006000200009

Foucault, M. (1983). The subject and power. En H. L. Dreyfus \& P. Rabinow (Eds.), Michel Foucault: Beyond structuralism and hermeneutics (pp. 208-226). Chicago, Illinois: The University of Chicago Press.

Fouce Fernández, J. G. (2009). Voluntariado social en el siglo XXI: ¿movimiento social o instrumento neoliberal? Intervención Psicosocial, 18(2), 177-190. Recuperado de http://www.redalyc.org/articulo.oa?id=179814021008

Garcés Montoya, A. (2010). De organizaciones a colectivos juveniles: panorama de la participación política juvenil. Última Década, 18(32), 61-83.

http://dx.doi.org/10.4067/S0718-22362010000100004

Montenegro, M. (2001). Conocimientos, agentes y articulaciones. Una mirada situada a la intervención social. Athenea Digital, (0). Recuperado de http://atheneadigital.net/article/view/n0-montenegro/17-html-es

Montero, M. (1984). La psicología comunitaria: orígenes, principios y fundamentos teóricos. Revista Latinoamericana de Psicología, 16(3), 387-400. Recuperado de http://www.redalyc.org/articulo.oa?id=80516303

Montero, M. (2003). Teoría y práctica en psicología comunitaria. La tensión entre comunidad y sociedad. Buenos Aires, Argentina: Paidós.

Montero, M. (2004). Introducción a la psicología comunitaria: desarrollos, conceptos y procesos. Buenos Aires, Argentina: Paidós.

Ornelas, J. (1997). Psicologia comunitária: Origens, fundamentos e áreas de intervenção. Análise Psicológica, 15(3), 375-388. Recuperado de http://www.scielo.mec.pt/scielo.php?script=sci_arttext\&pid=S0870-82311997000300002

Oxhorn, P. (2004). La paradoja del gobierno autoritario: organización de los sectores populares en los ochenta y promesa de inclusión. Política, 43, 57-83. Recuperado de http://www.redalyc.org/pdf/645/64504304.pdf 
Paredes, J. -P. (2013). Movilizarse tiene sentido: análisis cultural en el estudio de movilizaciones sociales. Psicoperspectivas, 12(2), 16-27.

http://dx.doi.org/10.5027/psicoperspectivas-Vol13-Issue2-fulltext-279

Pérez, C. (2002). Crisis de la familia y subjetividad pública en Chile hoy. En I. Piper (Comp.), Políticas, sujetos y resistencias: debates y críticas en psicología social (pp. 191-206). Santiago, Chile: Arcis.

Pérez Serrano, G. (1994). Investigación cualitativa. Retos e interrogantes: Vol. 1. Métodos. Madrid, España: La Muralla.

Pujal i Llomart, M. (2004). La identidad (el self). En T. Ibáñez García (Coord.), Introducción a la psicología social (pp. 93-138). Barcelona, España: Universidad Obrera de Cataluña.

Rodríguez Gómez, G., Gil Flores, J., \& García Jiménez, E. (1996). Metodología de la investigación cualitativa. Málaga, España: Aljibe.

Ruiz-Olabuénaga, J. (1996). Metodología de la investigación cualitativa. Bilbao, España: Universidad de Deusto.

Salamanca Castro, A. B. \& Martín-Crespo Blanco, M. C (2007). El muestreo en la investigación cualitativa. Nure Investigación, (27), 1-4. Recuperado de http://www.nureinvestigacion.es/OJS/index.php/nure/article/view/340/330

Salazar, C. A. \& Jaime, M. M. (2009). Participación en organizaciones sociales en Chile. ¿Una alternativa para mejorar el bienestar económico de los hogares? Estudios de Economía, 36(2), 191-215.

http://dx.doi.org/10.4067/S0718-52862009000200003

Scribano, A. \& De Sena, A. (2009). Construcción de conocimiento en Latinoamérica: algunas reflexiones desde la auto-etnografía como estrategia de investigación. Cinta de Moebio, (34), 1-15.

http://dx.doi.org/10.4067/S0717-554X2009000100001

Sisto Campos, V. (2004). Teoría(s) organizacional(es) postmoderna(s) y la gest(ac)ión del sujeto postmoderno. Athenea Digital, (6), 1-6. Recuperado de http://www.redalyc.org/articulo.oa?id=53700638

Stake, R. E. (1988). Investigación en estudio de casos. Madrid, España: Morata.

Ussher, M. M. (2006). El objeto de la psicología comunitaria desde el paradigma constructivista. Psicología para América Latina, (5). Recuperado de https://is.gd/yLmJCZ

Vázquez, F. (1994). Análisis de contenido categorial: el análisis temático. Barcelona, España: Universitat Autònoma de Barcelona.

Yopo, M. (2013). Individualización en Chile: individuo y sociedad en las transformaciones culturales recientes. Psicoperspectivas, 12(2), 4-15. Recuperado de http://www.scielo.cl/scielo.php?script=sci_arttext\&pid=S0718-69242013000200002 